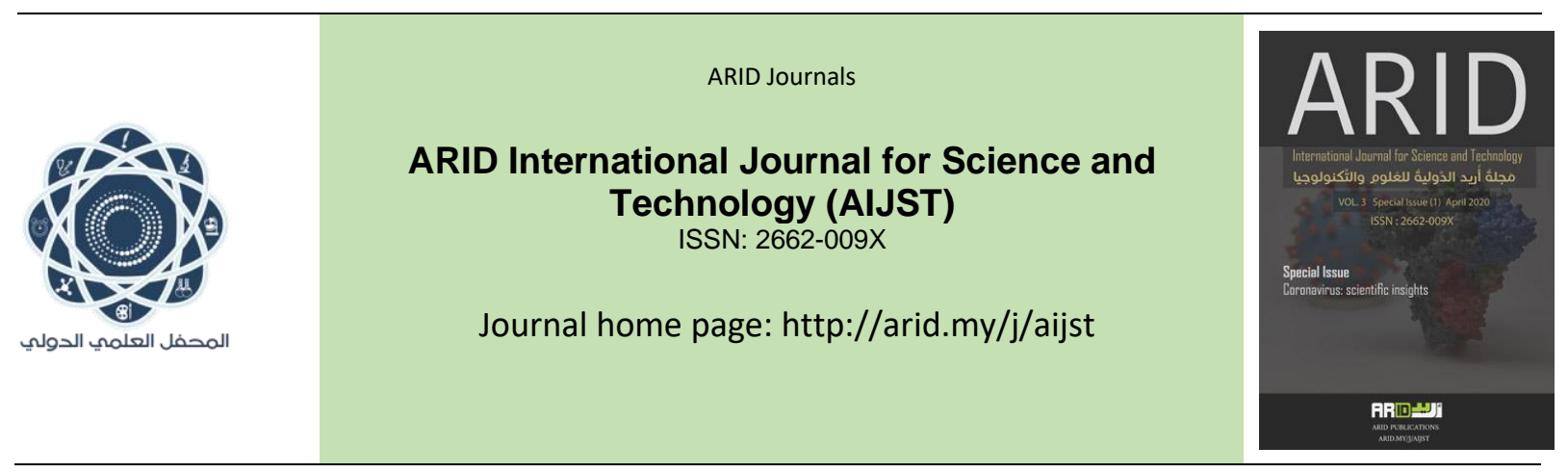

The use of passive immunity (Plasma) treatment for SARS-CoV-2 virus in

\title{
Basra, Iraq
}

Saad Shaheen Hammadi (1), Ali Raheem Hashim (1), Rafid Adil Abbood (1), Hassanein Mohammed Ali (2) Ali Salam Abdullah (2), Ali Radhi Kadhim (2), Amjed Maya Rodeen (2), Hiba Jabar Ashoor (2), , Shawqi Abdulsada Aziz (2), Basim Abdulkareem Abdulhassan (2), Mustafa Mawih (3)

(1) University of Basra

(2) Basra Health Directorate

(3) Kidney and Hypertension Center, Cincinnati, Ohio

$$
\begin{aligned}
& \text { استخدام العلاج بالمناعة السلبية (البلازما) لعلاج فايروس كورونا المستجد (كوفيد -19) في مدينة }
\end{aligned}
$$

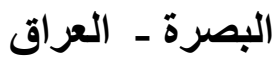

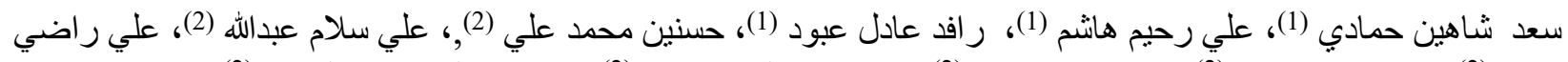

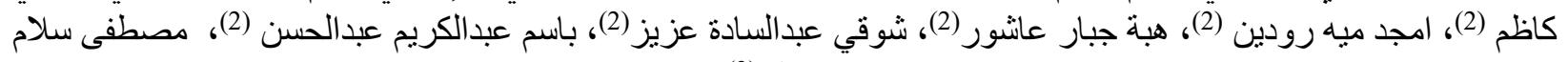

$$
\begin{aligned}
& \text { عبدالله (3) عبدال } \\
& \text { (1) (1) جامعة البصرة، (2) دائرة صحة البصرة، البصرة - العراق } \\
& \text { (3) مركز الكلى وارتفاع ضغط الدم في سينسيناتي أو هايو - الولايات المنحدة الامريكية }
\end{aligned}
$$

\section{President@uobasrah.edu.iq \\ arid.my/0004-6414}

https://doi.org/10.36772/arid.aijst.2020.312 


\begin{tabular}{l}
\hline A R T I C L E I N F O \\
\hline Article history: \\
Received 03/04/2020 \\
Received in revised form 20/04/2020 \\
Accepted 25/04/2020 \\
Available online $30 / 04 / 2020$ \\
\hline
\end{tabular}

\begin{abstract}
Reports from China and South Korea have shown improvements in critically ill Covid-19 patients using convalescent plasma from recovered individuals. On 25 March 2020, the Food and Drug Administration announced convalescent plasma guidelines for Covid-19 patients.
\end{abstract}

We collected plasma from one donor by a novel donation method that could safely provide plasma sufficient to 10 patients.

Four critically ill patients received convalescent plasma and had well-recognized clinical improvements in 12 to 72 hours.

To our knowledge, we are the first to report this novel convalescent plasma collection method. In the view of lack of enough donors, this method can be a breakthrough in the treatment of such a rapidly spreading illness. 


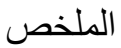

لقد أظهرت التقارير من الصين وكوريا الجنوبية تحسنا في مرضى كوفيد-19 ذوي الحالات الحرجة باستخدام بلازما النقاهة من الأشخاص المتعافين. كما أعلنت إدارة الغذاء و الدواء الأمريكية في 25 مارس / آذار 2020م إرشادات تخص بلازما النقاهة لمرضى كوفيد-19. تم استحصال بلازما النقاهة من متبر ع واحد، بطريقة تبرع مستحدثة والتي استطاعت أن توفر وبشكل آمن بلازما تكفي لعشرة مرضى.

استلم أربعة مرضى من ذوي الحالات الحرجة بلازما نقاهة، وقد حصل لايهم تحسناً سريرياً ملحوظاً خلال 12 إلى 72 ساعة. وبحسب معلومات الباحثين فإننا أول من يقدم تقرير ا باستخدام هذه الطريقة المستحدثة للتبرع ببلازما النقاهة. بالنظر إلى عدم وجود عدد كاف من المتبر عين، هذه الطريقة يمكنها أن تحدث فرقا في علاج مرض سريع الانتشار كهذا المرض. 


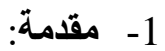

ما استخدمه الفريق الطبي ينطوي تحت إطار المناعة السلبية. و المقصود بالمناعة السلبية هو نقل أجسام مضادة أو خلايا تعمل بشكل متخصص ضد ذلك المرض فيما بعد، إن تكونت في الثخص الو اهب أو المتبرع نتيجة تعرضه إلى المرض بشكل طبيعي ( أحدث إصابة لهذا الشخص) أو عن طريق صناعي (اللقاح). كانت بدايات هذه الفكرة في آواخر القرن التاسع عشرعندمار راى "أميل فون بهرنك" وجماعته فعالية الأجسام المضادة لسموم بكتريا الخناق في إيقاف فعل هذا السم القاتل [1]. و أُعتبر استخدام هذه الأجسام المضادة لمعالجة المرضى بالأمر اض شديدة العدوى أهم إنجاز لعلم المناعة في القرن التاسع عشر [2-1]، إلا إن استخدام اللقاحات و المضادات الحياتية، قلل من استخدام هذه الطريقة لكونها كأي طريقة لابد أن يكون لها سلبيات و إيجابيات، وبقي استخدامها محدوداً، و لاز الت تستخدم مع بعض الحالات مثل فايروس داء الكلب وسموم الأفاعي و العقارب [3].

تم تصنيف فايروس كوفيد-19 المفاجى من المرتبة الر ابعة Class-4 في الخطورة، و هذه المرتبة تُمنح للمرض عندما يكون وبائياً وليس له لقاحاً و لا مضادا حياتيا [3-5]. كما أجبر المحافل الطبية للعودة بالتفكير بالمناعة السلبية ـ واستخدام أمصال المرضى الذين كتب لهم الثفاء من هذا المرض ليكون وسيلة للمعالجة . قام باحثُون من عدة دول بنقل أمصال المرضى المتبر عين لمعالجة مرضى كوفيد-19 [6-8]. كما قام فريق طبي مشترك من جامعة البصرة ودائرة صحة البصرة باستخدام العلاج بالمناعة السلبية (البلازما) لعلاج فيروس كورونا المستجد (كوفيد-19) و حققو انتائجاً ايجابية من خلال طبيبة شُفيت من الإصابة بالفايروس و التبر ع بالبلازما. ومن الأمور التي مهدت لنجاح هذه الطريقة هو توفر أجهزة فصل محتويات الدم Apheresis و التي تفصل مكونات الدم بشكل صحي و آمن

كانت المتبر عة طبيبة بعمر 58 سنة أصيبت بفايروس كوفيد-19 وشفيت وبعد مرور 14 يوما من الإصابة. عوملت المنبر عة معاملة أي شخص متبرع بالدم أو أحد مكوناته وذللك بإجر اء فحوصات نقل الدم كاملة. ب. أستخدام جهاز الـ Apheresis لفصل مكونات الدم 


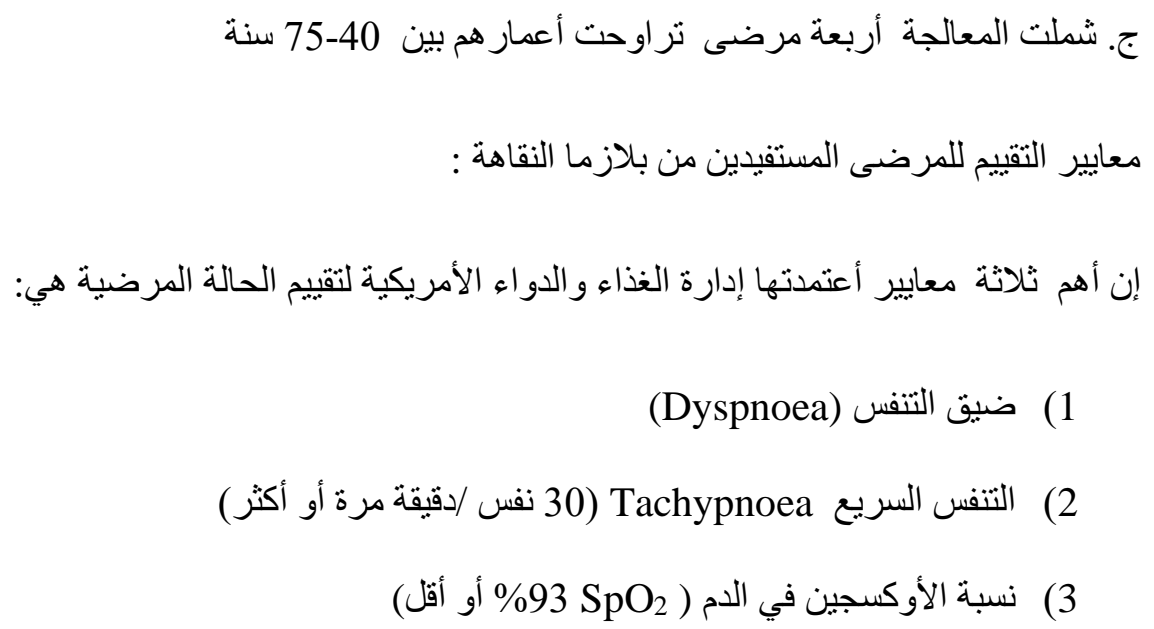

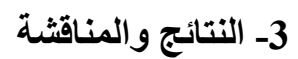

تم إعطاء بلازما النقاهة 250 مل لكل مريض، جميع المرضى كانو ا مشخصين بمرض كوفيد-19 بمسحة إيجابية وصورة طبقية إيجابية للصدر.

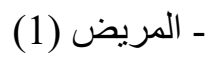

ذكر 40 عاماً، نسبة الأوكسجين في الدم 89 \% بدون أوكسجين مع انخفاض تدريجي يومي في نسبة الأوكسجين في الدم ، تسار ع في معدل التنفس ، وضيق التنفس . مدة بقائه في المستشفى للحظة إعطائه البلازما 7 أبام. بعد إعطاء البلازما كانت النتائج: بعد 12 ساعة، وُجدَ تحسناً ملحوظاً في حالة المريض العامة. بعد 24 ساعة، توقف انخفاض نسبة الأوكسجين في الدم وتحسن في التنفس و الحالة العامة. أما بعد 36 ساعة، نسبة الأوكسجين في الدم 90 \% وبدون ضخ الأوكسجين، المريض أصبح قادرا على التحرك و التجول بدون ضيث تنفس أو إعياء. بعد 48 ساعة، نسبة الأوكسجين في الدم 92 \% بدون أوكسجين. 


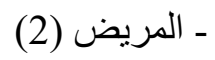

ذكر 75 عاماً، فترة بقائه في المستشفى للحظة إعطائه البلازما يومان، يرقد في العناية المركزة. نسبة الأوكسجين في الدم 84\% بدون ضخ أوكسجين ، يعاني من تسار ع في معدل التنفس وضيق تتفس. بعد إعطاء البلازما كانت النتائج:

بعد 12 ساعة، ارتفعت نسبة الأوكسين في الدم لتصبح 93\% بدون أوكسجين وتم نقله بعد 24 ساعة من العناية المركزة إلى الردهة الاعتيادية، و لا يعاني من ضيق في التنفس و لا تسارع في معدل التنفس.

بعد 36 ساعة نسبة الأوكسجين في الدم 93\% بدون أوكسجين، ولايعاني من ضيق في التنفس و لا تسارع في معدل التنفس. بعد 48 ساعة نسبة الأوكسجين في الدم 99\% بدون أوكسجين. وبدأ يشعر بالتحسن التام .

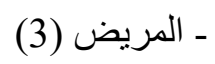

أنثى 48 عاماً، فترة بقائها في المستشفى قبل إعطائها البلازما 4 أيام، نسبة الأوكسجين في الدم 84\% بدون أوكسجين، تعاني من تسار ع في معدل التنفس وضيق تنفس شديد . بعد إعطاء البلازما كانت النتائج: بعد 12 ساعة : تحسن واضح في التنفس وارتفاع نسبة الأوكسجين في الدم إلى 95\% بدون أوكسجين. بعد 24 ساعة : نسبة الأوكسجين في الدم 91\% بدون أوكسجين. بعد 36 ساعة: نسبة الأوكسجين في الدم 93\% بدون أوكسجين. بعد 48 ساعة: نسبة الأوكسجين في الدم 98\% بدون أوكسجين.

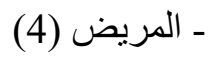


ذكر 70 عاماً، فترة بقائه في المستشفى قبل إعطائه البلازما 4 أيام، نسبة الأوكسجين في الدم 83\% بدون أوكسجين، يعاني من تسارع في معدل التنفس وضيق تنفس. لديه فثل مزمن في الكلى وفنش في القلب و السكري وارتفاع ضغط الدم و الوعي مشوش، يرقد في العناية المركزة. بعد إعطاء البلازما كانت النتائج : بعد 12 ساعة : نسبة الأوكسجين بالدم 85\% بدون أوكسجين وضيق تتفس أقل، مع تحسن في الوعي. بعد 24 ساعة : نسبة الأوكسجين في الدم 82\% بدون أوكسجين و 90-94\% مع الأوكسجين مع وعي مشوش. بعد 36 ساعة: نسبة الأوكسجين في الدم 91-92\% مع أوكسجين وو عي سليم، كما قلّ ضيق التنفس. بعد 48 ساعة : نسبة الأوكسجين في الدم 95\% مع أوكسجين والوعي والإدر اك سليمين و أقل اختناقا ، وتم نقله خارج العناية المركزة إلى الردهات الاعتيادية.

لقد نصت تعليمات إدارة الغذاء و الدواء الأمريكية المعلنة في 25 آذار/مارس 2020 و التي تم التعديل عليها في 8 نيسان/إبريل 2020، على أن في حال توفر أي من الصفات أعلاه لاى المريض المؤكد بالإصابة بفايروس كورونا المستجد/كوفيد 19 يستحق استلام البلازما من شخص قد تعافى من المرض بعد 14 يو ما من شفائه، و إنه يفضل أن يتم احتساب تركيز الأجسام المضادة في حال نوفر الفحص، وأوصت بتركيز لا يقل عن 80:1 [10] 4- الاستنتتاجات إن استخدام بلازما النقاهة يمكن أن يساهم في تسريع عملية شفاء المرضى أو يقلل/ يمنع مضاعفات المرض . 5- التوصيات:

حث وتثقيف المرضى المتعافين من المرض بالتبرع بالبلازما، للمساهمة في معالجة المرضى و هذا يتطلب من السلطات الصحية أن تقوم بالإعلان عن أن بلازما النقاهة يمكن إعطاءه للمرضى، لتشجيع التبرع وتشجيع الأطباء على استخدام البلازما كعلاج. شكر وتقدير يتقدم الباحثون بالثكر للزميلة أ.د عو اطف حميد عيسى - جامعة البصرة على مساعدتها في كتابة البحث و منابعة إرساله إلى مجلة "أُريد" الدولية للعلوم و التكنولوجيا 
FDA: Food and Drug Administration

SpO2: Saturated partial oxygen

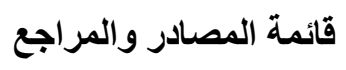

[1] Cf: Graham BS, Ambrosino DM. History of passive antibody administration for prevention and treatment of infectious diseases. Current Opinion in HIV and AIDS. 2015 May;10(3):129.

[2] G.Marano, S.Pupella , G.Facco, L.Catalano , G.M Liumbruno, G.Grazzini, “Convalescent plasma: new evidence for an old therapeutic tool? Blood Transfus." 2016 Mar;14(2):152-7. doi: 10.2450/2015.0131-15. Epub 2015 Nov 6. PMID: 26674811; PMCID: PMC4781783.

[3] S.J.Lucas,D.W.Barry and P. Kind , "Virus in immunodeficient mice, Infect Immun.” 1978 Apr; 20(1): 115-119. J Invest Dermatol. 1984 Jul;83(1 Suppl):121s-127s

[4] C. Huang, Y .Wang, X. Li, L .Ren, J. Zhao , Y .Hu , L .Zhang , G .Fan, J. Xu, X.Gu , Z.Cheng, T.Yu , J.Xia, Y.Wei , W.Wu , X.Xie , W.Yin, H.Li , M.Liu , Y.Xiao, H.Gao , L.Guo, J.Xie, G.Wang, R.Jiang, Z.Gao, Q.Jin , J.Wang, B.Cao. "Clinical features of patients infected with 2019 novel coronavirus in Wuhan, China, Lancet". 2020 Feb 15;395(10223):497-506. doi: 10.1016/S0140-6736(20)30183-5. Epub 2020 Jan 24. Erratum in: Lancet. 2020 Jan 30: PMID: 31986264; PMCID: PMC7159299.

[5] WHO. Novel coronavirus (COVID-19) situation. Updated March 24, 2020

[6] C.Shen, Z.Wang, F.Zhao, Y.Yang, J.Li, J.Yuan, F.Wang, D.Li, M.Yang, L.Xing, J.Wei, H.Xiao, Y.Yang, J.Qu, L.Qing, L.Chen, Z.Xu, L.Peng, Y.Li, H.Zheng, F.Chen,K. Huang, 
Y.Jiang, D.Liu, Z.Zhang, Y.Liu and L.Liu, "Treatment of 5 Critically Ill Patients WithCOVID-19 With Convalescent Plasma, JAMA"(2020), doi:10.1001/jama.2020.4783.

[7] Kai Duan, Bende Liu, Cesheng Li, Huajun Zhang, Ting Yu, Jieming Qu, Min Zhou, Li Chen, Shengli Meng, Yong Hu, Cheng Peng, Mingchao Yuan, Jinyan Huang, Zejun Wang, Jianhong Yu, Xiaoxiao Gao, Dan Wang, Xiaoqi Yu, Li Li, Jiayou Zhang, Xiao Wu, Bei Li, Yanping Xu, Wei Chen, Yan Peng, Yeqin Hu, Lianzhen Lin, Xuefei Liu, Shihe Huang, Zhijun Zhou, Lianghao Zhang, Yue Wang, Zhi Zhang, Kun Deng, Zhiwu Xia, Qin Gong, Wei Zhang, Xiaobei Zheng, Ying Liu, Huichuan Yang, Dongbo Zhou, Ding Yu, Jifeng Hou, Zhengli Shi, Saijuan Chen, Zhu Chen, Xinxin Zhang, Xiaoming Yang.” Effectiveness of convalescent plasma therapy in severe COVID-19 patients, National Academy of Sciences”,Apr.(2020),202004168;DOI:10.1073/pnas.2004168117.

[8] J.Y.Ahn, Y.Sohn, S. H.Lee, Y. Cho, J.H.Hyun, Y. J. Baek, S. J.Jeong, J. H.Kim, N. S.Ku, J. S. Yeom, J.Roh, M. Y. Ahn, B. S.Chin, Y. S.Kim, H. Lee, D.Yong, H. O. Kim, S.Kim and J.Y.Choi, "Use of Convalescent Plasma Therapy in Two COVID-19 Patients with Acute Respiratory Distress Syndrome in Korea. Journal of Korean medical science", 35(14),(2020)e149. https://doi.org/10.3346/jkms.2020.35.e149

[9] The American Society for Apheresis Clinical Guideline 2019.

[10] Investigational Covid-19 Convalescent Plasma Industrial Guide, U.S. Department of Health and Human Services Food and Drug Administration Center for Biologics Evaluation and Research April 2020 